\title{
An autoimmune cause of confusion in a patient with a background of hypothyroidism
}

\author{
Jonathan Brown ${ }^{1}$ and Luqman Sardar² \\ 1Brighton and Sussex University Hospitals NHS Trust and ${ }^{2}$ Elderly Care, Brighton and Sussex University Hospitals \\ NHS Trust, Brighton, UK
}

Correspondence should be addressed to J Brown

Email

jonathan.brown10@nhs.net

\section{Summary}

A 68-year-old previously independent woman presented multiple times to hospital over the course of 3 months with a history of intermittent weakness, vacant episodes, word finding difficulty and reduced cognition. She was initially diagnosed with a TIA, and later with a traumatic subarachnoid haemorrhage following a fall; however, despite resolution of the haemorrhage, symptoms were ongoing and continued to worsen. Confusion screen blood tests showed no cause for the ongoing symptoms. More specialised investigations, such as brain imaging, cerebrospinal fluid analysis, electroencephalogram and serology also gave no clear diagnosis. The patient had a background of hypothyroidism, with plasma thyroid function tests throughout showing normal free thyroxine and a mildly raised thyroid-stimulating hormone (TSH). However plasma anti-thyroid peroxidise (TPO) antibody titres were very high. After discussion with specialists, it was felt she may have a rare and poorly understood condition known as Hashimoto's encephalopathy (HE). After a trial with steroids, her symptoms dramatically improved and she was able to live independently again, something which would have been impossible at presentation.

\section{Learning points:}

- In cases of subacute onset confusion where most other diagnoses have already been excluded, testing for antithyroid antibodies can identify patients potentially suffering from HE.

- In these patients, and under the guidance of specialists, a trial of steroids can dramatically improve patient's symptoms.

- The majority of patients are euthyroid at the time of presentation, and so normal thyroid function tests should not prevent anti-thyroid antibodies being tested for.

- Due to high titres of anti-thyroid antibodies being found in a small percentage of the healthy population, HE should be treated as a diagnosis of exclusion, particularly as treatment with steroids may potentially worsen the outcome in other causes of confusion, such as infection.

\section{Background}

The case presented is of a rare disease, however, one where the correct treatment can greatly improve patients' symptoms, and therefore, we feel this diagnosis should be considered in similar presentations.

\section{Case presentation}

This case is of a 68 -year-old woman with a past medical history of hypothyroidism due to Hashimoto's thyroiditis (on levothyroxine), hypertension, hyperlipidaemia and recurrent sinusitis. She suffered from no other autoimmune condition. Her initial presentation was transient right arm weakness, right facial droop and slurred speech which lasted for 15 minutes before completely resolving. She was referred by her GP to the stroke clinic where, following a normal computed tomography (CT) scan of her head and normal ultrasound carotids, she was diagnosed with a left hemisphere transient ischaemic attack (TIA). Around a month later she presented to accident and emergency (A\&E) after being found collapsed. When found, she was 
displaying expressive dysphasia and was not recognising members of her family. A collateral history from her husband revealed for the previous 3 weeks she had been suffering from intermittent episodes of expressive dysphasia, short-term memory loss, vacant episodes (around 10 per day), tremor in both upper limbs and reduced concentration. CT head was inkeeping with an acute subarachnoid haemorrhage, which was confirmed on magnetic resonance imaging (MRI). It is possible that this was a traumatic haemorrhage due to the fall and that her preceding symptoms contributed to the fall itself; however, this is speculation and the sequence of events is difficult to establish. CT angiogram of the head showed no aneurysms, which was confirmed on cerebral catheter angiography. Her cognition during this admission was assessed using the Montreal Cognitive Assessment (MOCA), which gave a total score of $15 / 30$, with deficits primarily in visuospatial, attention and delayed recall tasks. She was later discharged, with the subarachnoid haemorrhage being the only diagnosis given during the admission, despite the collateral history of 3 weeks of symptoms prior to her fall. Symptoms continued, and several weeks later, she was again seen in A\&E and referred to stroke clinic due to confusion (being unable to work a door handle), expressive dysphasia and not recognising her husband. CT head was reported as normal. It was felt her symptoms may be related to seizure activity and so she was started on a trial of levetriacetam. The following week she once again attended A\&E due to another collapse and ongoing symptoms. At this point it was felt that her symptoms were unlikely to be explained by her previous intracranial haemorrhage or ischaemic strokes given her repeated brain imaging, and she was admitted for further investigations.

\section{Investigation}

Basic bloods were fairly unremarkable, with normal full blood count, inflammatory markers and renal function. Thyroid function tests (TFTs) showed a picture of subclinical hypothyroidism (TSH $6.04 \mathrm{mU} / \mathrm{L}$, free thyroxine $21.8 \mathrm{pmol} / \mathrm{L})$. This was later discussed with endocrinology who felt that these TFTs would not account for her symptoms. Electrocardiogram (ECG) showed sinus rhythm with 1st-degree heart block. CT head was once again normal. The working diagnosis at this point was epileptogenic events, and a plan was made of continue levetiracetam and arrange an electroencephalogram (EEG). On the advice of the neurology team, an MRI head and magnetic resonance angiogram (MRA) was also arranged. MRI showed haemosiderin deposition likely secondary to her recent subarachnoid haemorrhage, and no evidence of ischaemia. MRA head revealed no vascular abnormalities. EEG showed moderate excessive slow wave activity inkeeping with cerebrovascular disease but no focal abnormality or diagnostic epileptiform activity. However her symptoms were still ongoing, and so a lumbar puncture was performed. Cerebrospinal fluid (CSF) showed raised protein at $993 \mathrm{mg} / \mathrm{L}$ (with paired serum protein levels being borderline low) and raised immunoglobulin G (IgG) at $89 \mathrm{mg} / \mathrm{L}$ (paired serum IgG within normal range). Faint oligoclonal bands were seen in the CSF with an identical pattern in the serum. This was reported as being suggestive of systemic inflammation rather than intrathecal IgG synthesis. There was no bacterial growth on culture of the CSF, and screening for viral DNA using PCR was also negative. Other results from analysis of the CSF included no evidence of prion disease and normal total tau levels. Negative plasma serology included syphilis, human immunodeficiency virus (HIV), JC virus and measles.

It was considered that this could have represented an autoimmune encephalitis. However no plasma antibodies were detected against a large panel of nuclear antigens (to assess for anti-nuclear antibodies or ANA) including dsDNA and multiple extractable nuclear antigen (ENA) specificities. Anti-neutrophil cytoplasmic antibodies (ANCAs) of the c-ANCA and p-ANCA varieties were also assessed for in the plasma and were not found. Plasma voltage-gated potassium channel antibodies were also not detected. ESR was within normal limits.

The possibility of a paraneoplastic syndrome was also considered. However CT chest, abdomen and pelvis showed no evidence of malignancy. Flurodeoxyglucose positron emission tomography (FDG PET) showed mild, diffusely increased uptake within the thyroid gland. However, there was no suspicious FDG uptake to suggest the presence of a malignancy from the base of brain to mid-thigh.

The only investigation which did suggest a cause for her symptoms was an anti-TPO antibody titre of $>600$ IU/ $\mathrm{mL}$. Thyroid receptor antibody levels were $<0.4 \mathrm{u} / \mathrm{L}$. By this time she was under the care of the neurology team, who felt her condition may represent a condition known as steroid-responsive encephalopathy associated with autoimmune thyroiditis (SREAT), also known as Hashimoto's enecephalopathy. 


\section{Treatment}

She was started on methylprednisolone $500 \mathrm{mg}$ for 5 days and then switched to prednisolone. On this treatment her MOCA improved to 24/30 and was able to be discharged home, with her family reporting significant improvement in her symptoms.

\section{Outcome and follow-up}

On follow-up, she remained well with MOCA 28/30 back to her baseline. She was advised to reduce the steroids by $10 \mathrm{mg}$ per month and gradually wean off her levetiracetam.

\section{Discussion}

HE (also known as SREAT) is a rare condition associated with Hashimoto's thyroiditis. The cases described have a wide variety of clinical manifestations, including cognitive impairment, altered level of consciousness, seizures, tremor and myoclonus (1). A review in 2006 (2) found only 121 cases. One hospital-based study (3) screened patients for the disease and estimated the prevalence to be 2.1 cases per 100,000. Due to all the above, the condition can be very difficult to identify and is often misdiagnosed, as with our patient initially. The mean age of onset appears to be early 40s, and women are more commonly affected than men (4:1) (4). The defining features of the condition are raised plasma anti-thyroid antibodies and steroid responsiveness; however, anti-thyroid antibodies are raised in a small proportion of the healthy population, and therefore, some argue that the anti-thyroid antibodies have no direct pathological role, and instead, there may be another underlying autoimmune process (5), which is why some prefer the term SREAT (or doubt the validity of the syndrome at all (6)).

Pathogenesis is currently unclear. Symptoms of HE are not matched by symptoms of thyroiditis (most patients are euthyroid or show subclinical hypothyroidism at presentation (5)) and changes in plasma antibody titres do not correspond with neurological symptoms, suggesting plasma thyroid hormones or plasma anti-thyroid antibodies do not play a role. However several features, including being associated with high plasma antibody titres, steroid responsiveness and being more common in women, point towards an autoimmune component, and so far this appears to be the most investigated underlying pathology. As part of this, intrathecal antibodies have been assessed. The most common CSF abnormality found is raised protein, being found in $71.1 \%$ of patients in one review (6). Raised CSF IgG is also a reported abnormality. In one reported case (7), CSF IgG levels were found to be lower following treatment that resulted in clinical improvement, suggesting a role for CSF antibodies in the disease. Raised CSF protein and IgG were found in our patient; however, the response to therapy was not tested for. Intrathecal anti-thyroid antibodies have been an area of interest in several studies. In the review referenced earlier (6), anti-TPO antibodies were found in the CSF of $75 \%$ of patients with HE tested, and anti-thyroglobulin antibodies were found in the CSF of $84.6 \%$ of HE patients tested. Our patient unfortunately did not have these antibodies assessed for in her CSF, and plasma antibodies levels were not assessed after steroid therapy. One study (8) found anti-thyroid antibodies and circulating immune complexes in the CSF 6 patients with HE, but not in the CSF of 21 controls, again suggesting that intrathecal, rather than plasma anti-thyroid antibodies, may play a role. However titres were independent of the patient's clinical status or therapy. Furthermore no antigen has yet been found in the brain which binds to anti-thyroid antibodies. Other studies have attempted to find common antigens in brain and thyroid tissues which may fill in this missing link. One such suggested antigen is the amino (NH(2))- terminal of alpha-enolase (NAE), as antibodies against this have been found in patients with HE (9). Despite this, more evidence is needed to show a clear causal link between intrathecal antibodies and the disease, and by what mechanism these antibodies cause disease.

Management is usually corticosteroids, and the majority of patients respond to these (5), with symptoms improving or resolving over a few months. Other immunosupressants have been trialled, as well as IVIG and plasmapheresis. Other features of management include controlling seizures with anti-convulsants.

Due to its similar presentation to other conditions, the differential diagnosis is wide, including some conditions (such as infection) which may be worsened by treatment with corticosteroids. This, paired with the unclear pathogenesis of the disease, means these conditions should most definitely be treated as a diagnosis of exclusion, with it being essential to rule out other more common conditions before a diagnosis of $\mathrm{HE}$ is considered.

Declaration of interest

The authors declare that there is no conflict of interest that could be perceived as prejudicing the impartiality of the research reported. 


\section{Funding}

This research did not receive any specific grant from any funding agency in the public, commercial or not-for-profit sector.

\section{Patient consent}

Written consent has been obtained from the patient for publication of the submitted article.

\section{References}

1 de Holanda NC, de Lima DD, Cavalcanti TB, Lucena CS \& Bandeira F. Hashimoto's encephalopathy: systematic review of the literature and additional case. Journal of Neuropsychiatry and Clinical Neurosciences 201123 384-390. (https://doi.org/10.1176/jnp.23.4.jnp384)

2 Ferracci F \& Carnevale A. The neurological disorder associated with thyroid autoimmunity. Journal of Neurology 2006253 975-984. (https://doi.org/10.1007/s00415-006-0170-7)

3 Ferracci F, Bertiato G \& Moretto G. Hashimoto's encephalopathy: epidemiologic data and pathogenetic considerations. Journal of the
Neurological Sciences 2004217 165-168. (https://doi.org/10.1016/j. jns.2003.09.007)

4 www.uptodate.com/contents/hashimoto-encephalopathy

5 Chong JY, Rowland LP \& Utiger RD. Hashimoto encephalopathy: syndrome or myth? Archives of Neurology 200360 164-171. (https:// doi.org/10.1001/archneur.60.2.164)

6 Sunil GS \& Marish CN. Hashimoto's encephalitis. Journal of Clinical Endocrinology and Metabolism 200186 947. (https://doi.org/10.1210/ jcem.86.2.7241-4)

7 Gliebus G \& Lippa CF. Cerebrospinal immunoglobulin level changes and clinical response to treatment of Hashimoto's encephalopathy. American Journal of Alzheimer's Disease and Other Dementias 200924 373-376. (https://doi.org/10.1177/1533317509339162)

8 Ferracci F, Moretto G, Candeago RM, Cimini N, Conte F, Gentile M, Papa N \& Carnevale A. Antithyroid antibodies in the CSF: their role in the pathogenesis of Hashimoto's encephalopathy. Neurology 2003 60 712-714. (https://doi.org/10.1212/01.WNL.0000048660.71390. C6)

9 Yoneda M, Fujii A, Ito A, Yokoyama H, Nakagawa H \& Kuriyama M. High prevalence of serum autoantibodies against the amino terminal of alpha-enolase in Hashimoto's encephalopathy. Journal of Neuroimmunology 2007185 195-200. (https://doi.org/10.1016/j. jneuroim.2007.01.018)

Received in final form 26 March 2019

Accepted 8 April 2019 